\title{
Centers of Spatial and Time Inversion Symmetry in Magnetoelectric Crystalline Materials \\ Stephanie Gnewuch ${ }^{1}$, Efrain Rodriguez ${ }^{2}$ \\ ${ }^{1}$ University of Maryland, College Park ${ }^{2}$ University of Maryland \\ sgnewuch@umd.edu
}

Just as atomic structure dictates the properties of crystalline materials, the behavior of magnetic materials are dictated by their magnetic structure. Over the past several years we have researched antiferromagnetic materials in which the magnetic point group symmetry permits the formation of higher order toroidal moments. Since the magnetic moments in these candidate materials are ordered, this implies the toroidal moments would be ordered in a periodic structure as well. For example, if the arrangement of magnetic moments produces toroidal moments aligned along one direction in the crystal, the material would possess ferrotoroidal order.

After reviewing the candidates for ferrotoroidal order [1], we have undertaken several detailed neutron diffraction studies on members in the lithium transition metal phosphate series, including LiMPO4 (M=Co, Mn) [2] and the solid solution series LiMnxCo1-xPO4 and LiMnxFe1-xPO4. Previously at this conference, we discussed the evolution of the structure and magnetic moment in these series. All except LiMnPO4 were found to have the magnetic space group symmetry Pnma'. Based upon their tunability and magnetic symmetry, we concluded the entire series were candidate ferrotoroidic materials. In a subsequent year, we discussed in more detail the models for the toroidal moments in these and other candidate materials.

We have continued to study the structure and magnetism in this series with additional magnetization and diffraction experiments. We have also focused on understanding how the intersecting spatial and time inversion symmetry elements in the magnetic structure permit or forbid the ordering of toroidal moments. In this talk, I will present how having sites of -1' symmetry are crucial to modeling ordered toroidal moments, since both the magnetic and nonmagnetic atoms contribute to the formation of toroidal moments. Using the series of lithium transition metal phosphates studied, I will explain how different arrangements of toroidal moments can be classified as not only ferrotoroidal, but also antiferrotoroidal or non-toroidal.

[1] Gnewuch, S.; Rodriguez, E. J. Solid State Chem. 2019, 271, 175 - 190.

[2] Gnewuch, S.; Rodriguez, E. Inorg. Chem. 2020, 59, 5883-5895. 